International Electronic Journal of Geometry

Volume 7 No. 2 PP. 61-71 (2014) (CIEJG

\title{
THE DARBOUX TRIHEDRONS OF REGULAR CURVES ON A REGULAR SURFACE
}

\author{
EMRAH TUNÇ AND EMIN OZYILMAZ
}

(Communicated by Levent KULA)

\begin{abstract}
In this work, we study Differential Geometry of the curves on a regular surface in Euclidean space $R^{3}$ by using parameter curves which are not perpendicular to each other. The aim of this study is to investigate the formulas between the Darboux Vectors of the curve $(c)$, the parameter curve $\left(c_{1}\right)$ and the parameter curve $\left(c_{2}\right)$ which are not intersect perpendicularly.
\end{abstract}

\section{INTRODUCTION}

Roughly saying, geometry set out with the measurement of distances and angles. The geometry of curves in $R^{3}$ can be investigated by the topics which are general helices, involute-evolute curve couples, spherical curves and Bertrand curves. Such special curves are used many areas as seen computer graphics, topological combinatorics, especially, the modelling of cars, in physics and in some of real world problems like mechanical design or robotics.

In recent years the theory of degenerate submanifolds has been treated by researchers and some of the classical differential geometry topics have been extended to Lorentzian manifolds, [6]. In [6], authors had assumed that the parameter curves of any surface are orthognal. Moreover, some authors have aimed to determine the Frenet-Serret invariants in higher dimensions.

Another orthonormal frame on surface is the Darboux trihedrons. We use it to examine the special curves on surface as geodesic curves and asymptotic curves. By using the Darboux vector, various well-known formulas of differential geometry had been produced by $[1,4,5,2,3]$.

In this work, we study to investigate the formulas between the Darboux Vectors of the curve $(c)$, the parameter curves $\left(c_{1}\right)$ and $\left(c_{2}\right)$ which are not intersecting perpendicularly. Thus, we will find an opportunity to investigate regular surface by taking the parameter curves which are intersect under the angle $\theta$.

Date: Received: September 25, 2013 and Accepted: June 23, 2014.

2000 Mathematics Subject Classification. 53A04.

Key words and phrases. Classical Differential Geometry,Curves,Surfaces. 


\section{Preliminary}

To meet the requirements in the next sections, here, the basic elements of the theory of curves in the space $\mathbb{R}^{3}$ are briefly presented (A more complete elementary treatment can be found in [1]). The Euclidean space $\mathbb{R}^{3}$ provided with the standard flat metric given by

$$
<,>=d x_{1}^{2}+d x_{2}^{2}+d x_{3}^{2}
$$

where $\left(x_{1}, x_{2}, x_{3}\right)$ is a rectangular coordinate system of $\mathbb{R}^{3}$. Recall that, the norm of an arbitrary vector $a \in \mathbb{R}^{3}$ is given by $\|\vec{a}\|=\sqrt{\langle\vec{a}, \vec{a}\rangle}$. Let $\phi=\phi(s)$ be a regular curve in $\mathbb{R}^{3}$. $\phi$ is called an unit speed curve if the velocity vector $\vec{v}$ of $\phi$ satisfies $\|\vec{v}\|=1$. For the vectors $\vec{u}, \vec{w} \in \mathbb{R}^{3}$ it is said to be orthogonal if and only if $\langle\vec{u}, \vec{w}\rangle=0$. On the other hand, the vector $\vec{w}$ is called angular velocity vector of motion. If we consider any orthogonal trihedron as $\left\{\overrightarrow{e_{1}}, \overrightarrow{e_{2}}, \overrightarrow{e_{3}}\right\}$ we can write their derivative formulas as follows:

$$
\frac{d \overrightarrow{e_{1}}}{d t}=\vec{w} \wedge \overrightarrow{e_{i}}, \quad i=1,2,3
$$

where $\wedge$ is vectoral product, $[1]$.

Let we take a surface as $y=y(u, v)$. Denote by $\{\vec{t}, \vec{n}, \vec{b}\}$ the Frenet-Serret frame along the curve $(c)$ on $y=y(u, v)$. Another orthogonal frame on $y=y(u, v)$ is the Darboux trihedron as $\{\vec{t}, \vec{N}, \vec{g}\}$. For an arbitrary curve $(c)$ on surface, the orientation of the Darboux trihedron is written as

$$
\vec{t} \wedge \vec{N}=\vec{g}, \quad \vec{g} \wedge \vec{t}=\vec{N} \quad, \quad \vec{N} \wedge \vec{g}=\vec{t}
$$

and the Darboux vector of this trihedron is written as

$$
\vec{w}=\frac{\vec{t}}{T_{g}}+\frac{\vec{N}}{R_{g}}+\frac{\vec{g}}{R_{n}},
$$

where

$$
\begin{aligned}
& <\vec{t}, \vec{t}>=1, \quad<\vec{N}, \vec{N}>=1, \quad<\vec{g}, \vec{g}>=1, \\
& <\vec{t}, \vec{g}>=0, \quad<\vec{t}, \vec{N}>=0, \quad<\vec{g}, \vec{N}>=0 .
\end{aligned}
$$

where $\frac{1}{T_{g}}, \frac{1}{R_{n}}, \frac{1}{R_{g}}$ are geodesic torsion, normal curvature, geodesic curvature, and $T_{g}, R_{n}, R_{g}$, are radius of geodesic torsion ,radius of normal curvature, radius of geodesic curvature, respectively.

Also,the Darboux derivative formulas can be written as follows:

$$
\frac{d \vec{t}}{d s}=\vec{w} \wedge \vec{t}, \frac{d \vec{N}}{d s}=\vec{w} \wedge \vec{N}, \frac{d \vec{g}}{d s}=\vec{w} \wedge \vec{g} .
$$




\section{The Darboux Vector For The Darboux Trihedron Of A Regular Curve}

Let us express the parameter curves $u=$ const. as $\left(c_{1}\right)$ and $v=$ const. as $\left(c_{2}\right)$ which are constant on a regular surface $y=y(u, v)$. But, these curves are intersect under the angle $\theta$ (not perpendicular). Let any curve that is passing through a point $P$ on the surface be $(c)$. Let us take curves which are passing through the same point $P$ as $\left(c_{1}\right)$ and $\left(c_{2}\right)$. Let the unit tangent vectors of curves $(c),\left(c_{1}\right)$ and $\left(c_{2}\right)$ at the point $P$ be $\vec{t}, \overrightarrow{t_{1}}$ and $\overrightarrow{t_{2}}$, respectively. From [1], the edges of the Darboux trihedrons of parameter curves are

$$
\overrightarrow{t_{i}} \wedge \vec{N}=\overrightarrow{g_{i}}, \overrightarrow{g_{i}} \wedge \overrightarrow{t_{i}}=\vec{N}, \vec{N} \wedge \overrightarrow{g_{i}}=\overrightarrow{t_{i}},(i=1,2) .
$$

Here, the three Darboux trihedrons are written as below:

$$
[\vec{t}, \vec{N}, \vec{g}], \quad\left[\overrightarrow{t_{1}}, \vec{N}, \overrightarrow{g_{1}}\right], \quad\left[\overrightarrow{t_{2}}, \vec{N}, \overrightarrow{g_{2}}\right]
$$

Let the arc-lenght parameter of the curves $(c),\left(c_{1}\right)$ and $\left(c_{2}\right)$ be $s, s_{1}$ and $s_{2}$, respectively. Thus, we can write

$$
\begin{aligned}
& \overrightarrow{t_{1}}=\frac{\overrightarrow{r_{u}}}{\left\|\overrightarrow{r_{u}}\right\|}=\frac{\overrightarrow{u_{u}}}{\sqrt{E}}, \\
& \overrightarrow{t_{2}}=\frac{\overrightarrow{r_{v}}}{\left\|\overrightarrow{r_{v}}\right\|}=\frac{\overrightarrow{r_{v}}}{\sqrt{G}}, \\
& \vec{t}=\overrightarrow{r_{u}} \frac{d u}{d s}+\overrightarrow{r_{v}} \frac{d v}{d s} .
\end{aligned}
$$

Moreover,the parameter curves are intersect under the angle $\theta$.Thus, we have

$$
<\overrightarrow{t_{1}}, \overrightarrow{t_{2}}>=\cos \theta
$$

Then, the normal vector of surface is

$$
\vec{N}=\frac{\overrightarrow{t_{1}} \wedge \overrightarrow{t_{2}}}{\left\|\overrightarrow{t_{1}} \wedge \overrightarrow{t_{2}}\right\|}=\frac{\overrightarrow{t_{1}} \wedge \overrightarrow{t_{2}}}{\sin \theta}
$$

By considering the first two formulas of (3.2) in the third term,

$$
\vec{t}=\overrightarrow{r_{u}} \frac{d u}{d s}+\overrightarrow{r_{v}} \frac{d v}{d s}=\overrightarrow{t_{1}} \sqrt{E} \frac{d u}{d s}+\overrightarrow{t_{2}} \sqrt{G} \frac{d v}{d s}
$$

is written, [1]. On the other hand, let us consider the real angle between $\vec{t}$ and $\overrightarrow{t_{1}}$ as $\alpha$, and if we take inner product both side of (3.5) with $\overrightarrow{t_{1}}$ and $\overrightarrow{t_{2}}$ then

$$
\begin{gathered}
<\vec{t}, \overrightarrow{t_{1}}>=\cos \alpha=\sqrt{E} \frac{d u}{d s}+\cos \theta \sqrt{G} \frac{d v}{d s}, \\
<\vec{t}, \overrightarrow{t_{2}}>=\cos (\theta-\alpha)=\cos \theta \sqrt{E} \frac{d u}{d s}+\sqrt{G} \frac{d v}{d s}
\end{gathered}
$$


are obtained. Thus, from (3.6) and (3.7)

$$
\begin{aligned}
\frac{\sin (\theta-\alpha)}{\sin \theta} & =\sqrt{E} \frac{d u}{d s} \\
\frac{\sin \alpha}{\sin \theta} & =\sqrt{G} \frac{d v}{d s}
\end{aligned}
$$

are written. Finally, if we put (3.8) into (3.5), we have the following equation between the tangent vectors of the curves $(c),\left(c_{1}\right)$ and $\left(c_{2}\right)$ :

$$
\vec{t}=\frac{\sin (\theta-\alpha)}{\sin \theta} \overrightarrow{t_{1}}+\frac{\sin \alpha}{\sin \theta} \overrightarrow{t_{2}}
$$

Here, we shall denote the arc-elements $d s, d s_{1}$ and $d s_{2}$ of the parameter curves which are belongs to regular surface $y=y(u, v)$, and then we express as follows:

$$
\begin{array}{r}
d s^{2}=E d u^{2}+2 F d u d v+G d v^{2} \\
d s_{1}^{2}=E d u^{2} \\
d s_{2}^{2}=G d v^{2} .
\end{array}
$$

Thus, considering (3.8) and (3.10), we have

$$
\begin{array}{r}
\frac{\sin (\theta-\alpha)}{\sin \theta}=\sqrt{E} \frac{d u}{d s}=\frac{d s_{1}}{d s}, \\
\frac{\sin \alpha}{\sin \theta}=\sqrt{G} \frac{d v}{d s}=\frac{d s_{2}}{d s} .
\end{array}
$$

Corollary 3.1. The third elements $\vec{g}, \overrightarrow{g_{1}}$ and $\overrightarrow{g_{2}}$ of the Darboux trihedrons $[\vec{t}, \vec{N}, \vec{g}]$ , $\left[\overrightarrow{t_{1}}, \vec{N}, \overrightarrow{g_{1}}\right]$ and $\left[\overrightarrow{t_{2}}, \vec{N}, \overrightarrow{g_{2}}\right]$ are linear dependent.

Proof. If we substitute the equation (3.9) in the first equality of (2.3) and consider the Darboux trihedrons of $\left(c_{1}\right)$ and $\left(c_{2}\right)$ we have just obtain

$$
\vec{g}=\frac{\sin (\theta-\alpha)}{\sin \theta} \overrightarrow{g_{1}}+\frac{\sin \alpha}{\sin \theta} \overrightarrow{g_{2}} .
$$

Thus, we get the expression.

Theorem 3.1. The Darboux trihedrons $\left[\overrightarrow{t_{1}}, \vec{N}, \overrightarrow{g_{1}}\right]$ and $\left[\overrightarrow{t_{2}}, \vec{N}, \overrightarrow{g_{2}}\right]$ of the parameters curves $\left(c_{1}\right)$ and $\left(c_{2}\right)$ of the surface $y=y(u, v)$ are written by Darboux instantaneous vectors as follows:

$$
\frac{\partial \overrightarrow{t_{i}}}{d s}=\vec{w}_{i} \wedge \overrightarrow{t_{i}}, \frac{\partial \vec{N}}{d s}=\vec{w}_{i} \wedge \vec{N}, \frac{\partial \overrightarrow{g_{i}}}{d s}=\vec{w}_{i} \wedge \overrightarrow{g_{i}}, \quad(i, j=1,2) .
$$

Proof. If we consider the Darboux trihedrons $\left[\overrightarrow{t_{1}}, \vec{N}, \overrightarrow{g_{1}}\right]$ and $\left[\overrightarrow{t_{2}}, \vec{N}, \overrightarrow{g_{2}}\right]$ of parameter curves, we see that the normal vector $\vec{N}$ is coincide. Then, considering (3.4) 


$$
\begin{array}{r}
\overrightarrow{g_{1}}=\overrightarrow{t_{1}} \wedge \vec{N}=\overrightarrow{t_{1}} \wedge\left(\frac{\overrightarrow{t_{1}} \wedge \overrightarrow{t_{2}}}{\sin \theta}\right)=\frac{-\overrightarrow{t_{2}}\left(<\overrightarrow{t_{1}}, \overrightarrow{t_{1}}>\right)+\overrightarrow{t_{1}}\left(<\overrightarrow{t_{1}}, \overrightarrow{t_{2}}>\right)}{\sin \theta} \\
=\frac{\overrightarrow{t_{1}} \cos \theta-\overrightarrow{t_{2}}}{\sin \theta}, \\
\overrightarrow{g_{2}}=\overrightarrow{t_{2}} \wedge \vec{N}=\overrightarrow{t_{2}} \wedge\left(\frac{\overrightarrow{t_{1}} \wedge \overrightarrow{t_{2}}}{\sin \theta}\right)=\frac{-\overrightarrow{t_{2}}<\left(\overrightarrow{t_{1}}, \overrightarrow{t_{2}}\right)>+\overrightarrow{t_{1}}\left(<\overrightarrow{t_{2}}, \overrightarrow{t_{2}}>\right)}{\sin \theta} \overrightarrow{-\overrightarrow{t_{2}} \cos \theta+\overrightarrow{t_{1}}} \\
\sin \theta
\end{array}
$$

are obtained. From (2.2), we write

$$
\begin{aligned}
& \frac{\partial \overrightarrow{t_{1}}}{d s_{1}}=\overrightarrow{w_{1}} \wedge \overrightarrow{t_{1}}, \frac{\partial \vec{N}}{d s_{1}}=\overrightarrow{w_{1}} \wedge \vec{N}, \frac{\partial \overrightarrow{g_{1}}}{d s_{1}}=\overrightarrow{w_{1}} \wedge \overrightarrow{g_{1}}, \\
& \frac{\partial \overrightarrow{t_{2}}}{d s_{2}}=\overrightarrow{w_{2}} \wedge \overrightarrow{t_{2}}, \frac{\partial \vec{N}}{d s_{2}}=\overrightarrow{w_{2}} \wedge \vec{N}, \frac{\partial \overrightarrow{g_{2}}}{d s_{2}}=\overrightarrow{w_{2}} \wedge \overrightarrow{g_{2}} .
\end{aligned}
$$

If (3.14) is substituted in the third equality (3.16),we get

$$
\begin{gathered}
\frac{\partial \overrightarrow{g_{1}}}{\partial s_{1}}=\frac{\partial\left[\frac{1}{\sin \theta}\left(\cos \theta \overrightarrow{t_{1}}-\overrightarrow{t_{2}}\right)\right]}{\partial s_{1}}=\frac{1}{\sin \theta}\left(\frac{\partial \overrightarrow{t_{1}}}{\partial s_{1}} \cos \theta-\frac{\partial \overrightarrow{t_{2}}}{\partial s_{1}}\right)= \\
\frac{1}{\sin \theta}\left[\left(\overrightarrow{w_{1}} \wedge \overrightarrow{t_{1}}\right) \cos \theta-\frac{\partial \overrightarrow{t_{2}}}{d s_{1}}\right], \\
\frac{\partial \overrightarrow{g_{1}}}{\partial s_{1}}=\overrightarrow{w_{1}} \wedge \overrightarrow{g_{1}}=\overrightarrow{w_{1}} \wedge\left[\frac{1}{\sin \theta}\left(\cos \theta \overrightarrow{t_{1}}-\overrightarrow{t_{2}}\right)\right] \\
=\frac{1}{\sin \theta}\left(\left(\overrightarrow{w_{1}} \wedge \overrightarrow{t_{1}}\right) \cos \theta-\left(\overrightarrow{w_{1}} \wedge \overrightarrow{t_{2}}\right)\right) .
\end{gathered}
$$

Then, from (3.18) and (3.19), we have

$$
\frac{\partial \overrightarrow{t_{2}}}{d s_{1}}=\overrightarrow{w_{1}} \wedge \overrightarrow{t_{2}}
$$

Thus, the derivative of $\overrightarrow{t_{2}}$ with respect to $\overrightarrow{s_{1}}$ is written by the vectoral product of $\overrightarrow{w_{1}}$ and $\overrightarrow{t_{2}}$.

Similarly, it is easy to see that the other vectors can be written by the same metod.

Corollary 3.2. By using the vectors $\overrightarrow{t_{1}}, \overrightarrow{t_{2}}$ and $\vec{N}$, we can express $\vec{w}, \overrightarrow{w_{1}}$ and $\overrightarrow{w_{2}}$ as follows:

$$
\vec{w}=\frac{\overrightarrow{t_{1}}}{\sin \theta}\left[\frac{\sin (\theta-\alpha)}{T_{g}}+\frac{\cos (\theta-\alpha)}{R_{n}}\right]+\frac{\overrightarrow{t_{2}}}{\sin \theta}\left[\frac{\sin \alpha}{T_{g}}-\frac{\cos \alpha}{R_{n}}\right]+\frac{\vec{N}}{R_{g}},
$$




$$
\begin{aligned}
& \overrightarrow{w_{1}}=\overrightarrow{t_{1}}\left[\frac{1}{\left(T_{g}\right)_{1}}+\frac{\cos \theta}{\sin \theta\left(R_{n}\right)_{1}}\right]-\frac{\overrightarrow{t_{2}}}{\sin \theta\left(R_{n}\right)_{1}}+\frac{\vec{N}}{\left(R_{g}\right)_{1}}, \\
& \overrightarrow{w_{2}}=\frac{\overrightarrow{t_{1}}}{\sin \theta\left(R_{n}\right)_{2}}+\left[\frac{1}{\left(T_{g}\right)_{2}}-\frac{\cos \theta}{\sin \theta\left(R_{n}\right)_{2}}\right] \overrightarrow{t_{2}}+\frac{\vec{N}}{\left(R_{g}\right)_{2}} .
\end{aligned}
$$

Proof. From (2.4), we can write the Darboux vectors of the $[\vec{t}, \vec{N}, \vec{g}],\left[\overrightarrow{t_{1}}, \vec{N}, \overrightarrow{g_{1}}\right]$ and $\left[\overrightarrow{t_{2}}, \vec{N}, \overrightarrow{g_{2}}\right]$ as

$$
\begin{gathered}
\vec{w}=\frac{\vec{t}}{T_{g}}+\frac{\vec{N}}{R_{g}}+\frac{\vec{g}}{R_{n}}, \\
\overrightarrow{w_{1}}=\frac{\overrightarrow{t_{1}}}{\left(T_{g}\right)_{1}}+\frac{\vec{N}}{\left(R_{g}\right)_{1}}+\frac{\overrightarrow{g_{1}}}{\left(R_{n}\right)_{1}}, \\
\overrightarrow{w_{2}}=\frac{\overrightarrow{t_{2}}}{\left(T_{g}\right)_{2}}+\frac{\vec{N}}{\left(R_{g}\right)_{2}}+\frac{\overrightarrow{g_{2}}}{\left(R_{n}\right)_{2}} .
\end{gathered}
$$

Then, if we consider the equation(3.9),(3.14) and (3.15) according to the vectors $\overrightarrow{t_{1}}$ and $\overrightarrow{t_{2}}$ and substitute in (3.24), we get (3.21),(3.22) and (3.23).

Theorem 3.2. If we consider the tangent vectors $\overrightarrow{t_{1}}$ and $\overrightarrow{t_{2}}$ of the parameter curves $\left(c_{1}\right)$ and $\left(c_{2}\right)$ on the surface $y=y(u, v)$, then we obtain the following relations:

$$
\begin{aligned}
\text { i) }<\overrightarrow{t_{1}}, \frac{\partial \overrightarrow{t_{2}}}{\partial s_{1}}>=-<\overrightarrow{t_{2}}, \frac{\partial \overrightarrow{t_{1}}}{\partial s_{1}}>=\frac{(\sqrt{E})_{v}-\cos \theta(\sqrt{G})_{u}}{\sqrt{E G}}, \\
\text { ii) }<\overrightarrow{t_{2}}, \frac{\partial \overrightarrow{t_{1}}}{\partial s_{2}}>=-<\overrightarrow{t_{1}}, \frac{\partial \overrightarrow{t_{2}}}{\partial s_{2}}>=\frac{(\sqrt{G})_{u}-\cos \theta(\sqrt{E})_{v}}{\sqrt{E G}} .
\end{aligned}
$$

Proof. From (3.2), $\overrightarrow{t_{2}}=\frac{\overrightarrow{r_{u}}}{\sqrt{E}}$ and $\overrightarrow{t_{2}}=\frac{\overrightarrow{r_{v}}}{\sqrt{G}}$ are written. And also, we know that

$$
\begin{gathered}
<\overrightarrow{t_{1}}, \overrightarrow{t_{2}}>=\cos \theta \Rightarrow<\overrightarrow{r_{u}}, \overrightarrow{r_{v}}>=\cos \theta \sqrt{E} \sqrt{G}, \\
E=(\sqrt{E})^{2}={\overrightarrow{r_{u}}}^{2} \Rightarrow \sqrt{E}(\sqrt{E})_{v}=\overrightarrow{u_{u_{v}}} \overrightarrow{r_{u}}, \\
G=(\sqrt{G})^{2}={\overrightarrow{r_{v}}}^{2} \Rightarrow \sqrt{G}(\sqrt{G})_{u}=\overrightarrow{r_{v_{u}}} \overrightarrow{r_{v}} .
\end{gathered}
$$

By taking differential from $\overrightarrow{t_{1}}=\frac{\overrightarrow{r_{u}}}{\sqrt{E}}$ and $\overrightarrow{t_{2}}=\frac{\overrightarrow{r_{v}}}{\sqrt{G}}$, we obtain

$$
\begin{aligned}
& \frac{\partial \overrightarrow{t_{1}}}{\partial v}=\frac{\overrightarrow{r_{u}}(\sqrt{E})-(\sqrt{E})_{v} \overrightarrow{r_{u}}}{E}, \\
& \frac{\partial \overrightarrow{t_{2}}}{\partial u}=\frac{r_{u_{v}}\left(\sqrt{G}-(\sqrt{G})_{u} \overrightarrow{r_{v}}\right.}{G} .
\end{aligned}
$$

Thus, we write 
(3.30) $\left\langle\overrightarrow{t_{2}}, \frac{\partial \overrightarrow{t_{1}}}{\partial v}>=\frac{\overrightarrow{v_{v}}}{\sqrt{G}} \frac{\left(r_{u_{v}} \sqrt{E}-(\sqrt{E})_{v_{v}} \overrightarrow{r_{u}}\right.}{E}\right)=\frac{(\sqrt{G})_{u}-\cos \theta(\sqrt{E})_{v}}{\sqrt{E}}$,

(3.31) $\left\langle\overrightarrow{t_{1}}, \frac{\partial \overrightarrow{t_{2}}}{\partial u}\right\rangle=\frac{\overrightarrow{r_{u}}}{\sqrt{E}}\left(\frac{r_{u_{v}}(\sqrt{G})-(\sqrt{G})_{u} \overrightarrow{r_{v}}}{G}\right)=\frac{(\sqrt{E})_{v}-\cos \theta(\sqrt{G})_{u}}{\sqrt{G}}$.

On the other hand, we have

$$
\begin{aligned}
& \frac{\partial \overrightarrow{t_{1}}}{\partial s_{2}}=\frac{\partial \overrightarrow{t_{1}}}{\partial v} \frac{d v}{d s_{2}}=\frac{1}{\sqrt{G}} \frac{\partial \overrightarrow{t_{1}}}{\partial v} . \\
& \frac{\partial \overrightarrow{t_{2}}}{\partial s_{1}}=\frac{\partial \overrightarrow{t_{2}}}{\partial u} \frac{d u}{d s_{1}}=\frac{1}{\sqrt{E}} \frac{\partial \overrightarrow{t_{2}}}{\partial u} .
\end{aligned}
$$

Thus, taking inner product of (3.32) and (3.33) by the vector $\overrightarrow{t_{2}}$ and the vector $\overrightarrow{t_{1}}$ ,and considering (3.30)and(3.31), we have (3.26) and(3.25).

The other cases can be seen easily.

Corollary 3.3. If we take differential from $\left\langle\overrightarrow{t_{1}}, \overrightarrow{t_{2}}\right\rangle=\cos \theta$ with respect to $u$ and $v$, we get

$$
<\overrightarrow{t_{1}}, \frac{\partial \overrightarrow{t_{2}}}{\partial v}>=-<\overrightarrow{t_{2}}, \frac{\overrightarrow{t_{1}}}{\partial v}>=-\left[\frac{(\sqrt{G})_{u}-\cos \theta(\sqrt{E})_{v}}{\sqrt{E}}\right],
$$

The same holds for

$$
<\overrightarrow{t_{1}}, \frac{\partial \overrightarrow{t_{2}}}{\partial u}>=\frac{(\sqrt{E})_{v}-\cos \theta(\sqrt{G})_{u}}{\sqrt{G}}
$$

Thus, we have

$$
\begin{gathered}
\frac{\partial}{\partial v}\left(<\overrightarrow{t_{1}}, \frac{\partial \overrightarrow{t_{2}}}{\partial u}>-\frac{\partial}{\partial u}\left(<\overrightarrow{t_{1}}, \frac{\partial \overrightarrow{t_{2}}}{\partial v}>\right)=\right. \\
\frac{\partial}{\partial v}\left(\frac{(\sqrt{E})_{v}-\cos \theta(\sqrt{G})_{u}}{\sqrt{G}}\right)+\frac{\partial}{\partial u}\left(\frac{(\sqrt{G})_{u}-\cos \theta(\sqrt{E})_{v}}{\sqrt{E}}\right) .
\end{gathered}
$$

Theorem 3.3. The following geodesic curvature equalities are satisfied for the parameter curves $\left(c_{1}\right)$ and $\left(c_{2}\right)$

$$
\begin{aligned}
& \frac{1}{\left(R_{g}\right)_{1}}=\frac{1}{\sin \theta \sqrt{E G}}\left(\cos \theta(\sqrt{G})_{u}-(\sqrt{E})_{v}\right), \\
& \frac{1}{\left(R_{g}\right)_{2}}=\frac{1}{\sin \theta \sqrt{E G}}\left(\left(\sqrt{G}_{u}\right)-\cos \theta(\sqrt{E})_{v}\right) .
\end{aligned}
$$


Proof. i) From (3.25) and (3.20), we write

$$
\begin{gathered}
<\overrightarrow{t_{1}}, \frac{\partial \overrightarrow{t_{2}}}{\partial s_{1}}>=\left(\frac{(\sqrt{E})_{v}-\cos \theta(\sqrt{G})_{u}}{\sqrt{E G}}\right), \\
\frac{\partial \overrightarrow{t_{2}}}{\partial s_{1}}=\overrightarrow{w_{1}} \wedge \overrightarrow{t_{2}} .
\end{gathered}
$$

It follows that we have

$$
\frac{(\sqrt{E})_{v}-\cos \theta(\sqrt{G})_{u}}{\sqrt{E G}}=<\overrightarrow{t_{1}}, \overrightarrow{w_{1}} \wedge \overrightarrow{t_{2}}>=<\overrightarrow{w_{1}}, \overrightarrow{t_{2}} \wedge \overrightarrow{t_{1}}>=-\sin \theta<\vec{N}, \overrightarrow{w_{1}}>
$$

Then, from (3.24), if we take inner product both of side $\overrightarrow{w_{1}}$ with $-\sin \theta \vec{N}$ we obtain

$$
-\sin \theta<\vec{N}, \vec{w}_{1}>=-\frac{\sin \theta<\vec{N}, \vec{N}>}{\left(R_{g}\right)_{1}} \Rightarrow \vec{N} \vec{w}_{1}=\frac{1}{\left(R_{g}\right)_{1}} .
$$

Thus

$$
\frac{1}{\left(R_{g}\right)_{1}}=\frac{1}{\sin \theta \sqrt{E G}}\left(\cos \theta(\sqrt{G})_{u}-(\sqrt{E})_{v}\right)
$$

is obtained. Similarly, $(i i)$ can be proven.

Theorem 3.4. Let us consider any curve (c) on the surface and the arc elements of curves $(c),\left(c_{1}\right)$ and $\left(c_{2}\right)$ is $d s, d s_{1}$ and $d s_{2}$, respectively. Let the Darboux instantaneous rotation vectors of $\left(c_{1}\right)$ and $\left(c_{2}\right)$ be $\overrightarrow{w_{1}}, \overrightarrow{w_{2}}$ and if the real angle between the tangent $\vec{t}$ of curve $(c)$ and $\overrightarrow{t_{1}}$ is $\alpha$, then

$$
\left(\frac{\sin (\theta-\alpha)}{\sin \theta} \overrightarrow{w_{1}}+\frac{\sin \alpha}{\sin \theta} \overrightarrow{w_{2}}\right) \wedge \overrightarrow{t_{1}}=\vec{A} \wedge \overrightarrow{t_{1}}
$$

and

$$
\frac{d \overrightarrow{t_{1}}}{d s}=\vec{A} \wedge \overrightarrow{t_{1}}, \frac{d \overrightarrow{t_{2}}}{d s}=\vec{A} \wedge \overrightarrow{t_{2}}, \frac{d \vec{N}}{d s}=\vec{A} \wedge \vec{N}
$$

are satisfied.

Proof. If we consider (3.11) and (3.13), then

$$
\begin{gathered}
\frac{\partial \overrightarrow{t_{1}}}{\partial s}=\frac{\partial \overrightarrow{t_{1}}}{\partial s_{1}} \frac{d s_{1}}{d s}+\frac{\partial \overrightarrow{t_{1}}}{\partial s_{2}} \frac{d s_{2}}{d s}=\frac{\sin (\theta-\alpha)}{\sin \theta}\left(\overrightarrow{w_{1}} \wedge \overrightarrow{t_{1}}\right)+\frac{\sin \alpha}{\sin \theta}\left(\overrightarrow{w_{2}} \wedge \overrightarrow{t_{1}}\right)= \\
\left(\frac{\sin (\theta-\alpha)}{\sin \theta} \overrightarrow{w_{1}}+\frac{\sin \alpha}{\sin \theta} \overrightarrow{w_{2}}\right) \wedge \overrightarrow{t_{1}}=\vec{A} \wedge \overrightarrow{t_{1}}
\end{gathered}
$$

is obtained. Similarly, the others are satisfied.

Corollary 3.4. The following equality

$$
<\overrightarrow{t_{2}}, \frac{d \overrightarrow{t_{1}}}{d s}>=-<\overrightarrow{t_{1}}, \frac{d \overrightarrow{t_{2}}}{d s}>=\sin \theta<\vec{A}, N>
$$

is hold. 
Theorem 3.5. Let us consider the curves $(c),\left(c_{1}\right)$ and $\left(c_{2}\right)$ which are intersect a point $P$ on the surface $y=y(u, v)$. Let the Darboux instantaneous rotation vectors of these curves at the point $P$ be $\vec{w}, \overrightarrow{w_{1}}$ and $\overrightarrow{w_{2}}$, respectively. We obtain following equality between the Darboux vectors $\vec{w}, \overrightarrow{w_{1}}$ and $\overrightarrow{w_{2}}$ :

$$
\vec{w}=\frac{\sin (\theta-\alpha)}{\sin \theta} \overrightarrow{w_{1}}+\frac{\sin \alpha}{\sin \theta} \overrightarrow{w_{2}}+\vec{N} \frac{d \alpha}{d s} .
$$

Proof. From (3.9)

$$
\vec{t}=\frac{\sin (\theta-\alpha)}{\sin \theta} \overrightarrow{t_{1}}+\frac{\sin \alpha}{\sin \theta} \overrightarrow{t_{2}},
$$

can be written. Then, by taking derivatives with respect to $s$ from equation (3.43), we obtain

$$
\frac{d \vec{t}}{d s}=\frac{\sin (\theta-\alpha)}{\sin \theta} \frac{d \overrightarrow{t_{1}}}{d s}+\frac{\sin \alpha}{\sin \theta} \frac{d t_{2}}{d s}+\left[-\frac{\cos (\theta-\alpha)}{\sin \theta} \overrightarrow{t_{1}}+\frac{\cos \alpha}{\sin \theta} \overrightarrow{t_{2}}\right] \frac{d \alpha}{d s}
$$

On the other hand, considering the Darboux trihedrons $\left[\overrightarrow{t_{1}}, \vec{N}, \overrightarrow{g_{1}}\right]$ and $\left[\overrightarrow{t_{2}}, \vec{N}, \overrightarrow{g_{2}}\right]$, we write

$$
\overrightarrow{t_{1}}=\vec{N} \wedge \overrightarrow{g_{1}}, \quad \overrightarrow{t_{2}}=\vec{N} \wedge \overrightarrow{g_{2}}
$$

From (3.14) and (3.15), if $\overrightarrow{g_{1}}$ and $\overrightarrow{g_{2}}$ are substituted in (3.45) we obtain

$$
\overrightarrow{t_{1}}=\vec{N} \wedge \frac{1}{\sin \theta}\left(\cos \theta \overrightarrow{t_{1}}-\overrightarrow{t_{2}}\right), \quad \overrightarrow{t_{2}}=\vec{N} \wedge \frac{1}{\sin \theta}\left(-\cos \theta \overrightarrow{t_{2}}+\overrightarrow{t_{1}}\right)
$$

By substituting the equations (3.46) in (3.44), we have

$$
\begin{aligned}
(3.47) \frac{d \vec{t}}{d s}=\frac{\sin (\theta-\alpha)}{\sin \theta} \frac{d \overrightarrow{t_{1}}}{d s}+\frac{\sin \alpha}{\sin \theta} \frac{d t_{2}}{d s}+[ & -\frac{\cos (\theta-\alpha)}{\sin ^{2} \theta} N \wedge\left(\cos \theta \overrightarrow{t_{1}}-\overrightarrow{t_{2}}\right) \\
& \left.+\frac{\cos \alpha}{\sin ^{2} \theta} N \wedge\left(\overrightarrow{t_{1}}-\overrightarrow{t_{2}} \cos \theta\right)\right] \frac{d \alpha}{d s} .
\end{aligned}
$$

According to the theorem 4,

$$
\frac{d \overrightarrow{t_{1}}}{d s}=\vec{A} \wedge \overrightarrow{t_{1}}, \frac{\overrightarrow{t_{2}}}{d s}=\vec{A} \wedge \overrightarrow{t_{2}}, \vec{A}=\frac{\sin (\theta-\alpha)}{\sin \theta} \overrightarrow{w_{1}}+\frac{\sin \alpha}{\sin \theta} \overrightarrow{w_{2}},
$$

are known. And, by using the trigonometric expression, we find

$$
\begin{gathered}
\frac{d \vec{t}}{d s}=\frac{\sin (\theta-\alpha)}{\sin \theta} \vec{A} \wedge \overrightarrow{t_{1}}+\frac{\sin \alpha}{\sin \theta} \vec{A} \wedge \overrightarrow{t_{2}}+N \wedge\left[\frac{\sin (\theta-\alpha)}{\sin \theta} \overrightarrow{t_{1}}+\frac{\sin \alpha}{\sin \theta} \overrightarrow{t_{2}}\right] \frac{d \alpha}{d s} \\
=\vec{A} \wedge\left[\frac{\sin (\theta-\alpha)}{\sin \theta} \overrightarrow{t_{1}}+\frac{\sin \alpha}{\sin \theta} \overrightarrow{t_{2}}\right]+N \wedge\left[\frac{\sin (\theta-\alpha)}{\sin \theta} \overrightarrow{t_{1}}+\frac{\sin \alpha}{\sin \theta} \overrightarrow{t_{2}}\right] \frac{d \alpha}{d s} \\
=\vec{A} \wedge \vec{t}+(\vec{N} \wedge \vec{t}) \frac{d \alpha}{d s} \\
=\left[\vec{A}+\vec{N} \frac{d \alpha}{d s}\right] \wedge \vec{t} .
\end{gathered}
$$


Thus

$$
\frac{d \vec{t}}{d s}=\vec{k} \wedge \vec{t}
$$

where

$$
\vec{k}=\left[\vec{A}+\vec{N} \frac{d \alpha}{d s}\right]
$$

After that,

$$
\vec{A}=\vec{k}-\vec{N} \frac{d \alpha}{d s}
$$

is obtained. By writing (3.49) in the third expression of (3.40) we obtain

$$
\frac{d \vec{N}}{d s}=\vec{A} \wedge \vec{N}=\left(\vec{k}-\frac{d \alpha}{d s} \vec{N}\right) \wedge \vec{N}=\vec{k} \wedge \vec{N}
$$

Since $\vec{W}$ is the Darboux vector, we have

$$
\frac{d \vec{t}}{d s}=\vec{w} \wedge \vec{t}, \frac{d N}{d s}=\vec{w} \wedge \vec{N}
$$

Then, considering $(2.5),(3.47),(3.50)$ and (3.51)

$$
\begin{gathered}
\frac{d \vec{t}}{d s}=\vec{w} \wedge \vec{t}=\vec{k} \wedge \vec{t} \Rightarrow 0=\vec{k} \wedge \vec{t}-\vec{w} \wedge \vec{t}=(\vec{k}-\vec{w}) \wedge \vec{t} \\
(\vec{k}-\vec{w})=\lambda \vec{t}
\end{gathered}
$$

and

$$
\begin{gathered}
\frac{d \vec{N}}{d s}=\vec{w} \wedge \vec{N}=\vec{k} \wedge \vec{N} \Rightarrow 0=\vec{k} \wedge \vec{t}-\vec{w} \wedge \vec{N}=(\vec{k}-\vec{w}) \wedge \vec{N}, \\
\vec{k}-\vec{w}=\mu \vec{N},
\end{gathered}
$$

are written. At the end, if we make equal (3.52) to (3.53), we have

$$
\vec{k}-\vec{w}=\lambda \vec{t}=\mu \vec{N} \Rightarrow \lambda=\mu=0 .
$$

Finally, $\vec{k}-\vec{w}=\overrightarrow{0}$ can be written. Thus, we get the theorem.

\section{REFERENCES}

[1] Akbulut, F., Bir Yuzey Uzerindeki Egrilerin Darboux Vektorleri. Ege Univ. Faculty of Science, Izmir, 1983.

[2] Do Carmo, M.P. , Differential Geometry of Curves and Surfaces, Prentice-Hall, Englewood Cliffs, 1976.

[3] Klingenberg,W., A Course in Differential Geometry, Springer-Verlag, Berlin and New York, 178 p., 1978.

[4] Kuhnel,W., Differential Geometry Curves-Surfaces-Manifolds, Eiesbaden, Braunchweig, 1999. 
[5] Milman,M.S and Parker,G.D., Elements of Differential Geometry,Prentice-Hall Inc,Englewood Cliffs, New Jersey, 1977.

[6] Ugurlu, H. and Caliskan, A., The Space-like and Time-like Surface Geometry by the Darboux Vector, Celal Bayar Univ., Manisa, 2012.

Department of Mathematics, Faculty of Science, University of Ege, Bornova, Izmir, TURKEY

E-mail address: emrahtunc@hotmail.com

Department of Mathematics, Faculty of Science, University of Ege, Bornova, Izmir, TURKEY

E-mail address: ozyilmaz@ege.edu.tr 